\title{
Innovative therapy concepts for pediatric brain tumors
}

\author{
Amedeo A. Azizi · Johannes Gojo • Andreas Peyrl (D)
}

Received: 2 May 2021 / Accepted: 9 July 2021 / Published online: 19 August 2021

(C) The Author(s) 2021

\begin{abstract}
Summary In recent years, novel insight into molecular mechanisms has allowed the identification of drug targets for various pediatric brain tumors. The aim of this article is to give an overview of new treatment options in neurofibromatosis type 1 (NF1), novel tyrosine kinase inhibitors that target oncogenic gene fusions in pediatric brain tumors, and antiangiogenesis as promising therapy especially in recurrent medulloblastoma.
\end{abstract}

Keywords NF1 - BRAF - Oncogenic fusion proteins Antiangiogenesis

\section{Introduction}

The survival rates of pediatric brain tumor patients improved constantly during the end of the $20^{\text {th }}$ century. This was mainly due to technological advances in imaging, neurosurgery and radiotherapy, as well as the use of combination chemotherapy. In the last two decades, however, outcome rates remained rather static, reflecting our lack of knowledge of the underlying molecular mechanisms of different pediatric brain tumors.

In recent years, molecular characterization has massively changed the landscape of pediatric central nervous system (CNS) malignancies [1]. This novel insight into molecular mechanisms also allows the identification of drug targets in different pediatric brain tumors. It is therefore realistic that the near future will bring further advancement in the treatment of these malignancies.

\footnotetext{
A. A. Azizi · J. Gojo · A. Peyrl ( $\square)$

Department of Pediatrics, Comprehensive Center for

Pediatrics, Medical University of Vienna, Waehringer Guertel

18-20, 1090 Vienna, Austria

andreas.peyrl@meduniwien.ac.at
}

\section{Targeting the pathway in low grade glioma by MEK inhibition}

Neurofibromatosis type 1 (NF1) is the most common genetic tumor predisposition syndrome associated with the development of CNS tumors with an incidence of 1 in 3000. In this monogenetic disease, the NF1 gene and consecutively the tumor suppressor neurofibromin (as regulator of RAS) is altered, leading to uncontrolled RAS-mitogen-activated protein kinase (RAS/MAPK) pathway activation upon damage of the second allele (second hit). Low grade gliomas (LGG) are the most frequent CNS malignancies associated with NF1 and are almost exclusively driven by this mutation [2]. Molecular analysis of sporadic LGGs has revealed that they also unanimously harbor alterations in the MAPK pathway [3].

While LGG is associated with a generally high overall survival rate and many patients can be cured by surgery alone, other LGGs necessitate nonsurgical treatment, the gold standard being chemotherapy with carboplatin and vincristine [4]. Some patients with LGG suffer multiple relapses and response to cytotoxic therapy diminishes with each progression [5]. Therefore, alternative therapy options are needed.

BRAF and MEK inhibitors were developed for the treatment of BRAF-mutated malignant melanoma. The MEK inhibitor selumetinib was evaluated in a pediatric trial for inoperable NF1-associated plexiform neurofibroma and demonstrated high volumetric response rates with tolerable side effects [6, 7]. The use of the first generation BRAF inhibitor sorafenib in LGG was associated with rapid tumor progression, finally proven to be caused by a positive feedback loop in cell signaling [8]. Hence consecutively, downstream inhibition by MEK inhibitors was attempted. Selumetinib was proven to be effective in a phase 1 trial and a more comprehensive phase 2 trial could underline these re- 
sults $[9,10]$. Prolonged progression-free survival (PFS) rates could be achieved, and in optic pathway glioma, improved visual outcomes were reported. Specifically, in the NF1 subgroup, a high partial response rate of $36 \%$ ( $60 \%$ stable disease) was observed with a $96 \%$ 2-year PFS. This can be considered as a class effect of MEK inhibitors, as similar results have been shown in patients treated with trametinib [11]. Yet, the efficacy of these agents remains to be proven in a first-line setting regarding long-term effects and side effects compared to standard of care chemotherapy. Such trials have already opened or will do so in the near future (e.g., LOGGIC Europe. ACNS1831). Possible side effects of MEK inhibition include frequently skin toxicities (in about $60 \%$ of patients) like acne or paronychia that are most often manageable by local therapies, and less often fatigue, gastrointestinal symptoms, CK elevation as well as rare cases of retinal detachment or left ventricular dysfunction [12-14]. BRAF-V600E-mutated pediatric LGG may not be as chemotherapy sensitive as other LGG [15]. The combination of the selective BRAF V600E inhibitor dabrafenib and the MEK inhibitor trametinib were proven effective in this subset of patients [16].

\section{Novel tyrosine kinase inhibitors target oncogenic gene fusions}

Apart from oncogenic signaling activation by loss of NF1, also somatic alterations, most prominently mutations or fusions involving BRAF, have been described in pediatric high-grade gliomas [17]. More recently, fusions of other signaling molecules, such as NTRK1/2/3, ALK, MET, or ROS1, have been discovered by large-scale high-throughput screens [18-20]. Importantly, as alterations in these oncogenes are also found in adult cancer types, inhibitors targeting these receptors have already undergone clinical development involving the pediatric population [21]. Preclinical analyses of our group and others demonstrate good effects of these inhibitors against fusionpositive cancer cells corroborating first results within ongoing clinical trials as well as selected case reports [19, 20, 22].

Currently available inhibitors include crizotinib, larotrectinib and entrectinib, which are approved by European Medicines Agency (EMA) and US Food and Drug Administration (FDA), as well as the secondgeneration inhibitors selitrectinib and repotrectinib, which are in clinical development [21]. It has to be considered that these inhibitors show different specificity for the aforementioned therapy targets and are only approved within certain indications, in particular in the pediatric population (Table 1).

Only a minor proportion of high-grade gliomas harbor these specific gene fusions, underlying that comprehensive molecular profiling of pediatric brain tumors at an early timepoint is required in order to facilitate detection of these therapeutic targets. With respect to brain tumors, penetration of the blood-brain barrier is also of particular interest. In general, penetration of crizotinib to the central nervous system is considered to be poor, but still efficacy in pediatric brain tumors has been described [19]. For the newer compounds larotrectinib and entrectinib both have demonstrated effects against pediatric brain tumors in a clinical setting $[20,22]$. These novel compounds are generally well tolerated, only a minority of patients require dose modifications or discontinuation of treatment [21]. With respect to blood-brain barrier penetration, first analyses point towards a more favorable profile of entrectinib, as animal studies have demonstrated higher penetration of tumor tissue [23]. In addition, it was recently shown that entrectinib also efficiently enters the cerebrospinal fluid [22]. Still, it has to be considered that safety profiles in the pediatric population, and in young children in particular, are currently more established for larotrectinib, which is also reflected by the approval independent of age. Response to these inhibitors is usually quick and frequently also for a prolonged period of time [21, 22]. Still, various resistance mechanisms have been described in the adult population, including resistance mutations, and second-generation inhibitors are already being developed. Less is known about resistance mechanisms in pediatric brain tumors, but we have recently described NF2 mutation and activation insulin receptor (INSR) signaling as potential resistance mechanisms [23]. The main ongoing pediatric trials with targeted drugs are listed in Table 2.

\section{Antiangiogenic treatment}

Medulloblastoma is a highly malignant embryonal tumor arising in the cerebellum. By using multimodal therapy that includes surgical resection, craniospinal irradiation and combination chemotherapy, approximately $70 \%$ of children achieve long-term survival, but with sometimes devastating sequelae [24].

\begin{tabular}{ll|l} 
Table 1 & Targeted inhibitors for oncogenic gene fusions in pediatric brain tumors (adapted from [17]) \\
\hline $\begin{array}{l}\text { Inhibitor } \\
\text { Crizotinib }\end{array}$ & Targets & Approval \\
\hline Larotrectinib & NTRK1/2/3 & ALK/ROS1 altered (lung cancer) \\
\hline Entrectinib & ALK, NTRK1/2/3, ROS1 & NTRK fusion-positive tumors (any age) \\
\hline $\begin{array}{l}\text { Selitrectinib } \\
\text { Repotrectinib }\end{array}$ & NTRK1/2/3 (including most mutations) & NTRK fusion-positive tumors (>12 years), ROS1 altered (lung cancer) \\
\hline & ALK, ROS1, NTRK1/2/3 (including most mutations) & - \\
\hline
\end{tabular}


Table 2 Main ongoing pediatric trials with targeted drugs

\begin{tabular}{ll|l|}
\hline Drug & Condition or disease & Identifier \\
\hline Selumetinib & Low-Grade Glioma (LGG) & NCT04166409 \\
\hline Mirdametinib & Recurrent or Progressive Low-Grade Glioma (LGG) & NCTO4576117 \\
\hline Trametinib, Everolimus & Low-Grade Glioma (LGG) & NCTO4923126 \\
\hline Trametinib & Recurrent Low-Grade Glioma (LGG) & NCTO4485559 \\
\hline Trametinib, Dabrafenib & Pediatric Glioma or Plexiform Neurofibroma and Activation of the MAPK/ERK Pathway & NCT03363217 \\
\hline Larotrectinib & Recurrent or Progressive Glioma in Children and Young Adults & NCT04201457 \\
\hline Larotrectinib & Untreated TRK Fusion Solid Tumors and TRK Fusion Relapsed Acute Leukemia & NCT03834961 \\
\hline Larotrectinib & High-Grade Glioma With NTRK Fusion & NCT04655404 \\
\hline Entrectinib & Tumors with NTRK-fusion in Children & NCT02637687 \\
\hline Selitrectinib & Locally Advanced or Metastatic Solid or Primary CNS Tumors & NCT02650401 \\
\hline Repotrectinib & Solid Tumors Harboring NTRK Fusion & NCT03215511 \\
\hline
\end{tabular}

In recent years, tremendous progress in molecular genetics has provided significant advancements in our understanding of medulloblastoma. There is now consensus on four distinct molecular subgroups: wingless (WNT), sonic hedgehog (SHH), group 3, and group 4 [25]. Recently, further subgroup heterogeneity has been defined [26]. These molecular groups have already formed the basis of the current clinical treatment protocol PNET5 (ClinicalTrials.gov Identifier: NCT02066220), where WNT-medulloblastomas are treated with reduced radio- and chemotherapy.

Despite the favorable outcome after the initial diagnosis, patients with relapsed medulloblastoma have a very poor prognosis, whether treated with conventional chemotherapy, high-dose chemotherapy with stem cell rescue, irradiation or combinations of these modalities $[27,28]$.

Angiogenesis, the formation of new blood vessels, is an important component of normal physiological processes such as wound healing. Moreover, tumor growth and metastases depend on the formation of new blood vessels [29].

The frequent delivery of different drugs, referred to as metronomic or antiangiogenic therapy, targets the endothelial cells while reducing the toxicity associated with standard dose chemotherapy. Antiangiogenic therapy inhibits vascular formation, thereby inhibiting tumor progression indirectly [30, 30].

Various trials have demonstrated that antiangiogenic drugs are generally insufficient as single agents, a combination of different agents is considered to be more promising [31].

We used an antiangiogenic multidrug regime, consisting of bevacizumab, thalidomide, celecoxib, fenofibrate, etoposide, and cyclophosphamide. All of these drugs have been shown to have antiangiogenic effects [32-37]. Additionally, we administered intraventricular therapy (etoposide and cytarabine), since the cerebrospinal fluid (CSF) compartment is not targeted with antiangiogenic therapy. With this regime, we achieved promising results, especially in recurrent medulloblastoma [38]. Three of the seven patients with recurrent medulloblastoma in our series achieved long-term survival [38]. Therapy was generally well tolerated, and toxicities were manageable. The most common toxicities were hypertension, a well-described side effect of bevacizumab, chemical arachnoiditis occurring after intraventricular liposomal cytarabine, and the patients were more prone to infections because of the immunosuppression. [38].

Our results suggested that this antiangiogenic drug combination may be beneficial for patients with recurrent medulloblastoma. Further investigation with a formal phase II study is in progress (Metronomic and Targeted Anti-angiogenesis Therapy for Children with Recurrent/Progressive Medulloblastoma (MEMMAT); ClinicalTrials.gov Identifier: NCT01356290).

\section{Conclusion}

The information of the genomic mechanisms has revolutionized our understanding of the underlying pathogenesis of pediatric brain tumors. Oncogenic gene fusions offer novel therapeutic opportunities for pediatric brain tumors and have already entered clinical practice. Open questions remain regarding the optimal timepoint of treatment initialization, duration of treatment, combination with other treatment modalities and avoidance of resistance. The laboratory-based findings will define a path for future clinical studies. The main challenge will be to design protocols with international collaboration that include molecular information, to improve the survival rate while minimizing toxicities.

\section{Take-home message}

- Targeting the BRAF pathway in inoperable NF1-associated tumors is a promising option.

- Oncogenic gene fusions offer novel therapeutic opportunities.

- Antiangiogenic combinations may be beneficial for recurrent medulloblastoma. 
Funding Open access funding provided by Medical University of Vienna.

Conflict of interest A.A. Azizi, J. Gojo and A. Peyrl declare that they have no competing interests.

Open Access This article is licensed under a Creative Commons Attribution 4.0 International License, which permits use, sharing, adaptation, distribution and reproduction in any medium or format, as long as you give appropriate credit to the original author(s) and the source, provide a link to the Creative Commons licence, and indicate if changes were made. The images or other third party material in this article are included in the article's Creative Commons licence, unless indicated otherwise in a credit line to the material. If material is not included in the article's Creative Commons licence and your intended use is not permitted by statutory regulation or exceeds the permitted use, you will need to obtain permission directly from the copyright holder. To view a copy of this licence, visit http://creativecommons.org/licenses/by/4.0/.

\section{References}

1. Capper D, Jones DTW, Sill M, et al. DNA methylation-based classification of central nervous system tumours. Nature. 2018;555(7697):469-74.

2. Fisher MJ, Jones DTW, Li Y, et al. Integrated molecular and clinical analysis of low-grade gliomas in children with neurofibromatosis type 1 (NF1). Acta Neuropathol. 2021;141(4):605-17.

3. Ryall S, Tabori U, Hawkins C. Pediatric low-grade glioma in the era of molecular diagnostics. Acta Neuropathol Commun. 2020;8(1):30.

4. Gnekow AK, Kandels D, Tilburg CV, et al. SIOP-E-BTG and GPOH guidelines for diagnosis and treatment of children and adolescents with low grade glioma. Klin Padiatr. 2019;231(3):107-35.

5. Kandels D, Pietsch T, Bison B, et al. Loss of efficacy of subsequent nonsurgical therapy after primary treatment failure in pediatric low-grade glioma patients-report from the German SIOP-LGG 2004 cohort. Int J Cancer. 2020;147(12):3471-89.

6. Gross AM, Wolters PL, Dombi E, et al. Selumetinib in children with inoperable plexiform neurofibromas. NEngl JMed. 2020;382(15):1430-42.

7. Dombi E, Baldwin A, Marcus LJ, et al. Activity of selumetinib in neurofibromatosis type 1-related plexiform neurofibromas. NEnglJ Med. 2016;375(26):2550-60.

8. Karajannis MA, Legault G, Fisher MJ, et al. Phase II study of sorafenib in children with recurrent or progressive lowgrade astrocytomas. Neuro Oncol. 2014;16(10):1408-16.

9. Banerjee A, Jakacki RI, Onar-Thomas A, et al. A phase I trial of the MEK inhibitor selumetinib (AZD6244) in pediatric patients with recurrent or refractory low-grade glioma: a pediatric brain tumor consortium (PBTC) study. Neuro Oncol. 2017;19(8):1135-44.

10. Fangusaro J, Onar-Thomas A, Poussaint TY, et al. A phase 2 trial of aelumetinib in children with recurrent optic pathway and hypothalamic low-grade glioma without NF1: a pediatric brain tumor consortium study. Neuro Oncol. 2021; https://doi.org/10.1093/neuonc/noab047.

11. Selt F, van Tilburg CM, Bison B, et al. Response to trametinib treatment in progressive pediatric low-grade glioma patients. J Neurooncol. 2020;149(3):499-510.

12. Campagne O, Yeo KK, Fangusaro J, et al. Clinical pharmacokinetics and pharmacodynamics of selumetinib. Clin Pharmacokinet. 2021;60(3):283-303.
13. Baldo F, Grasso AG, Cortellazzo Wiel L, et al. Selumetinib in the treatment of symptomatic intractable plexiform neurofibromas in neurofibromatosis type 1: a prospective case series with emphasis on side effects. Paediatr Drugs. 2020;22(4):417-23.

14. Avery RA, Trimboli-Heidler C, Kilburn LB. Separation of outer retinal layers secondary to selumetinib. J AAPOS. 2016;20(3):268-71.

15. Lassaletta A, Zapotocky M, Mistry M, et al. Therapeutic and prognostic implications of BRAF V600E in pediatric lowgrade gliomas. JClin Oncol. 2017;35(25):2934-41.

16. Hargrave DR, Bouffet E, Tabori U, et al. Efficacy and safety of dabrafenib in pediatric patients with BRAF V600 mutation-positive relapsed or refractory low-grade glioma: results from a phase I/IIa study. Clin Cancer Res. 2019;25(24):7303-11.

17. MackayA, Burford A, Carvalho D, et al. Integrated molecular meta-analysis of 1,000 pediatric high-grade and diffuse intrinsic pontine glioma. Cancer Cell. 2017;32(4):520-37.

18. International Cancer Genome Consortium PedBrain Tumor Project. Recurrent MET fusion genes represent a drug target in pediatric glioblastoma. Nat Med. 2016;22(11):1314-20.

19. Clarke M, Mackay A, Ismer B, et al. Infant high-grade gliomas comprise multiple subgroups characterized by novel targetable gene fusions and favorable outcomes. Cancer Discov. 2020;10(7):942-63.

20. Drilon A. TRK inhibitors in TRK fusion-positive cancers. Ann Oncol. 2019;30(8):viii23-viii30.

21. Mayr L, Guntner A, Madlener S, et al. Cerebrospinal fluid penetration and combination therapy of entrectinib for disseminated ROS1 / NTRK-fusion positive pediatric highgrade glioma. J Pers Med. 2020;10:290.

22. Fischer H, Ullah M, de la Cruz C, et al. Entrectinib, a TRK/ROS1 inhibitor with anti-CNS tumor activity: differentiation from other inhibitors in its class due to weak interaction with P-glycoprotein. Neuro-Oncology. 2020;22(6):819-29.

23. Lannering B, Rutkowski S, Doz F, et al. Hyperfractionated versus conventional radiotherapy followed by chemotherapy in standard-risk medulloblastoma: results from the randomized multicenter HIT-SIOP PNET 4 trial. J Clin Oncol. 2012;30(26):3187-93.

24. Northcott PA, Korshunov A, Pfister SM, et al. The clinical implications of medulloblastoma subgroups. Nat Rev Neurol. 2012;8(6):340-51.

25. Sharma T, Schwalbe EC, Williamson D, et al. Secondgeneration molecular subgrouping of medulloblastoma: an international meta-analysis of group 3 and group 4 subtypes. Acta Neuropathol. 2019;138(2):309-26.

26. Pizer B, Donachie PH, Robinson K, et al. Treatment of recurrent central nervous system primitive neuroectodermal tumours in children and adolescents: results of a children's cancer and leukaemia group study. Eur J Cancer. 2011;47(9):1389-97.

27. SabelM, FleischhackG, TippeltS, etal. Relapsepatterns and outcome after relapse in standard risk medulloblastoma: a report from the HIT-SIOP-PNET4 study. J Neurooncol. 2016;129(3):515-24.

28. Folkman J. Tumor angiogenesis: therapeutic implications. NEngl J Med. 1971;285(21):1182-6.

29. Browder T, Butterfield CE, Kräling BM, et al. Antiangiogenic scheduling of chemotherapy improves efficacy against experimental drug-resistant cancer. Cancer Res. 2000;60(7):1878-86. 


\section{short review}

30. Gillies RJ, Verduzco D, Gatenby RA. Evolutionary dynamics of carcinogenesis and why targeted therapy does not work. Nat Rev Cancer. 2012;12(7):487-93.

31. Leahy KM, Ornberg RL, Wang Y, et al. Cyclooxygenase-2 inhibition by celecoxib reduces proliferation and induces apoptosis in angiogenic endothelial cells in vivo. Cancer Res. 2002;62(3):625-31.

32. Panigrahy D, Kaipainen A, Butterfield CE, et al. Inhibition of tumor angiogenesis by oral etoposide. Exp Ther Med. 2010;1(5):739-46.

33. PanigrahyD, KaipainenA, HuangS, etal. PPARalphaagonist fenofibrate suppresses tumor growth through direct and indirect angiogenesis inhibition. Proc Natl Acad Sci U S A. 2008;105(3):985-90.

34. Willett CG, Boucher Y, di Tomaso E, et al. Direct evidence that the VEGF-specific antibody bevacizumab has antivascular effects in human rectal cancer. Nat Med. 2004;10(2):145-7.

35. Garcia AA, Hirte H, Fleming G, et al. Phase II clinical trial of bevacizumab and low-dose metronomic oral cyclophosphamide in recurrent ovarian cancer: a trial of the California, Chicago, and Princess Margaret Hospital phase II consortia. JClin Oncol. 2008;26(1):76-82.
36. D’Amato RJ, Loughnan MS, Flynn E, Folkman J. Thalidomide is an inhibitor of angiogenesis. Proc Natl Acad Sci U SA. 1994;91(9):4082-5.

37. Peyrl A, Chocholous M, Kieran MW, et al. Antiangiogenic metronomic therapy for children with recurrent embryonal brain tumors. Pediatr Blood Cancer. 2012;59(3):511-7.

38. Slavc I, Peyrl A, Gojo J, et al. Recurrent medulloblastoma-long-term survival with a "MEMMAT"-based antiangiogenic approach. Neuro-Oncology. 2020;22(3):397.

Publisher's Note Springer Nature remains neutral with regard to jurisdictional claims in published maps and institutional affiliations.

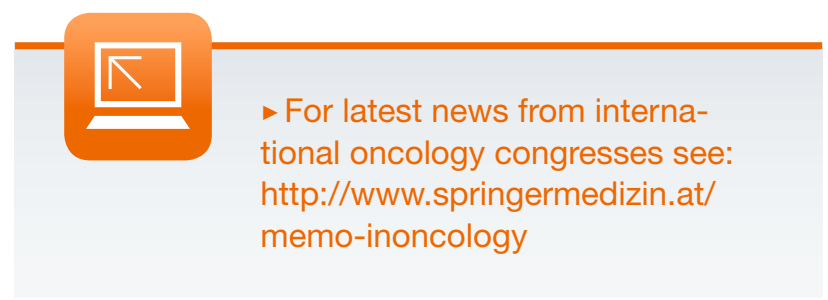

